\title{
Perspective Piece Application of the Relationship-Based Model to Engagement for Field Trials of Genetically Engineered Malaria Vectors
}

\author{
Ana Kormos, ${ }^{1 *}$ Gregory C. Lanzaro, ${ }^{1}$ Ethan Bier, ${ }^{2,3}$ George Dimopoulos, ${ }^{4}$ John M. Marshall, ${ }^{5,6}$ João Pinto, ${ }^{7}$ \\ Adionilde Aguiar dos Santos, ${ }^{8}$ Affane Bacar, ${ }^{9}$ Herodes Sousa Pontes Sacramento Rompão, ${ }^{10}$ and Anthony A. James ${ }^{11,12}$ \\ ${ }^{1}$ Vector Genetics Laboratory, University of California, Davis, California; ${ }^{2}$ Section of Cell and Developmental Biology, University of California, San \\ Diego, California; ${ }^{3}$ Tata Institute for Genetics and Society (TIGS)-UCSD, San Diego, California; ${ }^{4}$ Department of Molecular Microbiology and \\ Immunology, Malaria Research Institute (JHMRI), Bloomberg School of Public Health, Johns Hopkins University, Baltimore, Maryland; ${ }^{5}$ Division of \\ Epidemiology and Biostatistics, School of Public Health, University of California, Berkeley, California; ${ }^{6}$ Innovative Genomics Institute, Berkeley, \\ California; ${ }^{7}$ Global Health and Tropical Medicine, Instituto de Higiene e Medicina Tropical, Universidade Nova de Lisboa, Lisbon, Portugal; \\ ${ }^{8}$ Ministério da Saúde, Delegacia de Saúde Distrital de Água Grande, Cidade de São Tomé, São Tomé and Príncipe; ${ }^{9}$ Ministry of Health, Programme \\ Nationale de Lutte Contre le Paludisme, Moroni, Union of the Comoros; ${ }^{10}$ Ministério da Saúde, Programa Nacional de Luta Contra o Paludismo, \\ Cidade de São Tomé, São Tomé and Príncipe; ${ }^{11}$ Department of Microbiology and Molecular Genetics, University of California, Irvine, California; \\ ${ }^{12}$ Department of Molecular Biology and Biochemistry, University of California, Irvine, California
}

\begin{abstract}
The transition of new technologies for public health from laboratory to field is accompanied by a broadening scope of engagement challenges. Recent developments of vector control strategies involving genetically engineered mosquitoes with gene drives to assist in the eradication of malaria have drawn significant attention. Notably, questions have arisen surrounding community and regulatory engagement activities and of the need for examples of models or frameworks that can be applied to guide engagement. A relationship-based model (RBM) provides a framework that places stakeholders and community members at the center of decision-making processes, rather than as recipients of predetermined strategies, methods, and definitions. Successful RBM application in the transformation of healthcare delivery has demonstrated the importance of open dialogue and relationship development in establishing an environment where individuals are actively engaged in decision-making processes regarding their health. Although guidelines and recommendations for engagement for gene drives have recently been described, we argue here that communities and stakeholders should lead the planning, development, and implementation phases of engagement. The RBM provides a new approach to the development of ethical, transparent, and effective engagement strategies for malaria control programs.
\end{abstract}

\section{INTRODUCTION}

Engagement is a critical and challenging component in the science of public health. The work of promoting health and wellness, disease prevention, detection and response, and development of novel strategies to prevent infectious diseases requires multiple communication methods and an environment that facilitates open dialogue and exchange of knowledge, perspectives, and preferences by diverse groups of people.

In 2006, the NIH began emphasizing translational research, which included a community engagement (CE) component. ${ }^{1}$ Community engagement has become increasingly viewed as the keystone to translational science. ${ }^{2}$ Emerging technologies like gene drives for public health have accelerated the need for effective CE practices and credible, independent risk assessment to ensure safe, ethical, and scientifically rigorous trials of the technologies. Unlike drug or vaccine development, no industry standard yet exists to evaluate gene drive vector control methods, ${ }^{3}$ and the scope and impact of these methods may differ greatly suggesting that a different set of research ethics and considerations will be required.

There is an existing body of knowledge on engagement practices across many public health sectors, ${ }^{4-12}$ including recommendations specific to the testing of novel technologies for vector control..$^{8,13-15}$ All of these recommendations state

\footnotetext{
* Address correspondence to Ana Kormos, Department of Pathology, Microbiology and Immunology, University of California, Davis, 1089 Veterinary Medicine Dr., Davis, CA 95616. E-mail: akormos@
} ucdavis.edu (or imply) the importance of communication and development of trusting relationships in successful engagement activities. Although there are existing foundations on which to build an engagement program, attention has been drawn to specific questions surrounding the testing and introduction of new gene drive technologies. These include scale of application, participation and consent, existing regulatory and safety mechanisms, and long-term effects and accountability. Novel technologies present novel questions about best practices for engagement. Genetically engineered mosquitoes (GEMs) with gene drive offer the benefit of providing sustainable, long-term vector control over large areas without requiring individual behavior modification, or bias related to socioeconomic determinants. Although this offers obvious advantages, it also presents challenges, which include individual participation/ involvement in decision-making and the determination of definitions related to engagement (e.g., communities, approval, acceptance, and consent). Additional challenges include lack of existing regulation, ethical standards, and policies related to a technology that has the potential to affect human communities over large geographic areas which may increase in scale over time. Changing public perceptions and attitudes about science and technology, and the increasing expectation that communities should be involved early in the decision-making surrounding the science continue to drive the evolution of engagement strategies.

We consider here the application of a relationship-based model (RBM) for community and regulatory engagement processes designed specifically to support new gene drive vector control technologies. Engagement strategies often are developed and initiated by outside experts and academics 
working to support specific research programs and goals. ${ }^{16}$ The RBM is unique in that it provides a framework that supports decentralized decision-making and emphasizes the importance of stakeholder and community member leadership in the development and implementation of community and regulatory engagement strategies, definitions, and decisions (Figure 1). We believe that an approach for field trials of GEMs with gene drive for malaria control requires engagement and regulatory strategies that reflect the important biological and social dynamics unique to the place and population where the technology may be applied.

Definitions. We define engagement as the facilitation and support of collaboration, open communication, and dialogue for the exchange of knowledge, perspectives, opinions, and preferences among diverse groups of people. We apply this definition for audiences (communities, stakeholders, and publics) outlined by the National Academies of Sciences, Engineering, and Medicine ${ }^{13}$ and thus also reference the definitions they have provided for these groups (Box 1).

We define regulatory engagement as the engagement process with relevant stakeholders, community members, and publics who will be involved in the assessment of risk and regulatory oversight of the technology at the field trial site. The regulatory engagement process applies to the development of the risk assessment processes and new regulatory frameworks/pathways required for activities related to testing the gene drive technology.

\section{RELATIONSHIP-BASED MODEL, CONTEXT, AND CONCEPTS}

Relationship-based models, developed for transforming healthcare delivery, have evolved from a "patient-centered" concept that was originally elaborated in $1969 .{ }^{17}$ There have been many conceptualizations described since then, using different terms including relationship-centered care and person-centered care. The relationship-based care model emerged in $2004^{18}$ and is widely known for providing a framework and concepts designed to focus on the specific needs of the patient and family. In 2006, relationship-centered care described a similar framework expanding the focus to the community and has since been adapted for successful population health management strategies. ${ }^{19}$

An RBM provides applications for building and strengthening relationships and establishes an environment in which individuals are actively engaged in decision-making processes regarding their health and how they receive care, rather than as recipients of messaging campaigns and prescriptive guidelines. Relationship-based models recognize that the variable public health system can positively affect or influence personal habits, choices, options, and understanding and require collaborative, trusting relationships. Population health outcomes and acceptance of public health interventions are ultimately controlled by the decisions, values, beliefs, and circumstances of unique individuals who do not respond in a "one-size-fits-all" environment.

Focusing on the value of relationships has resulted in successful, innovative health systems that have shown improved population and community health outcomes. ${ }^{20-26}$ An expanded relationship between health delivery systems and the community promotes support of larger health improvement initiatives and traditional public health responsibilities of disease control and prevention that can be designed to meet the specific needs of the community/population. The WHO applied this concept to malaria, recently reporting that "conditions and opportunities must be created to support the coproduction of health in a way that places people at the center of malaria eradication considerations," and that if engagement

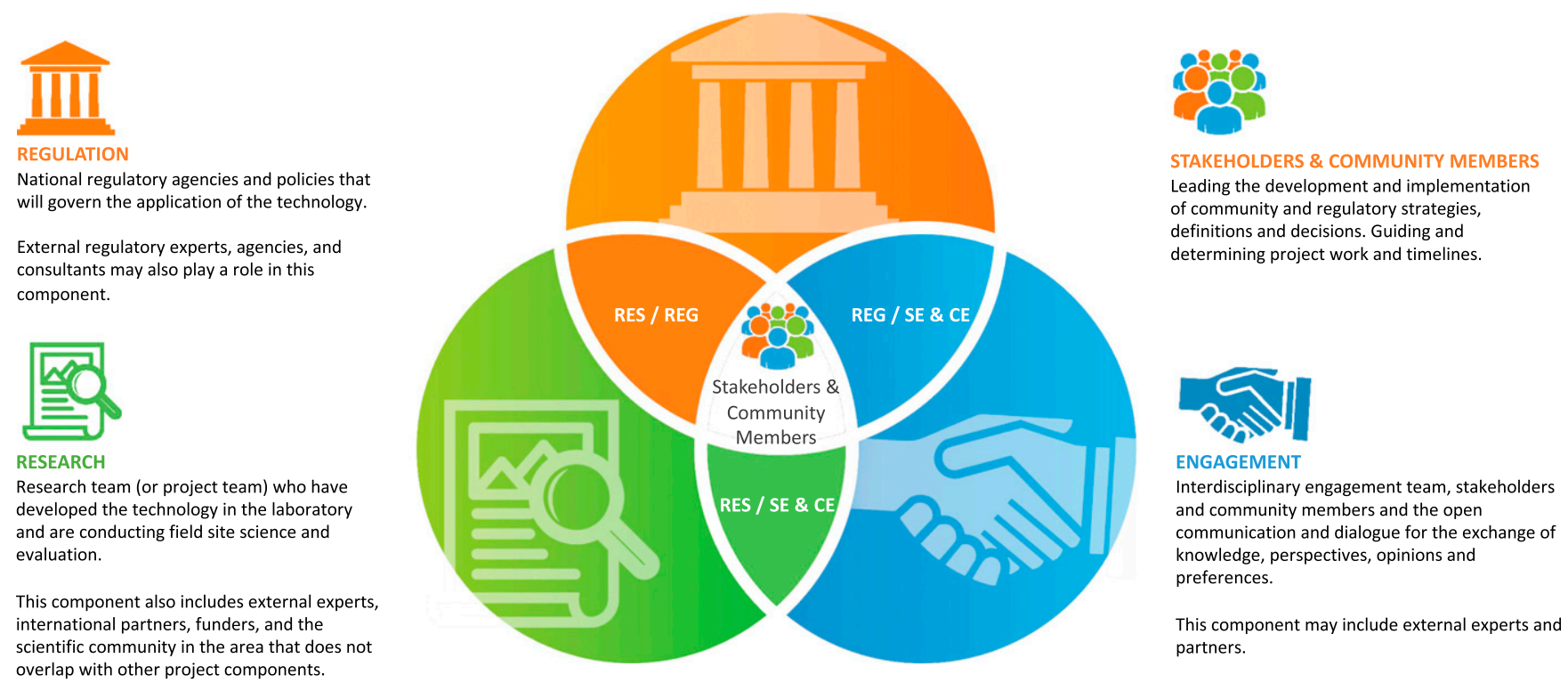

FIGURE 1. Relationship-based model (RBM) for community and regulatory engagement. Venn diagram showing interactions among key components applying a RBM. Stakeholders and community members are at the center and drive decision-making processes. Research (RES)/ Regulation (REG): Dialogue among researchers and regulators is critical in the assessment of risk, application of the technology, and monitoring and surveillance. REG/Stakeholder and Community Engagement (SE \& CE): Dialogue among regulators, stakeholders, and community members informs and guides the risk assessment and regulatory development processes, monitoring, and surveillance. RES/SE \& CE: Dialogue among researchers, stakeholders, and community will direct the scientific research and timelines and will determine if, when, where, and how the technology is applied. 
Box 1

Engagement audience definitions: Definitions as provided in Gene Drives on the Horizon: Advancing Science, Navigating Uncertainty, and Aligning Research with Public Values, published by the National Academies of Sciences, Engineering, and Medicine ${ }^{13}$

1. Communities: Groups of people who live within the geographical location or biologically relevant proximity to a potential site where research is taking place or where field releases may take place such that they have tangible and immediate interests in the research project. Communities are included within the broader category of "stakeholders."

2. Stakeholders: Organizations, groups, or persons with professional or personal interests sufficient to justify engagement, but who may, or may not, have geographic proximity to potential intervention sites for the research project.

3. Publics: Groups who lack the direct connection to a project that stakeholders and communities have but nonetheless have interests, concerns, hopes, fears, and values that can contribute and influence decision-making about the research and possible use of the vector control intervention.

for malaria control is to be successful, "one particularly important part of the broader process of engagement is the interaction between communities at risk of malaria and the health system with which they need to collaborate for a successful outcome."16 The RBM provides a salient framework for developing an effective, collaborative engagement strategy for malaria eradication programs.

The University of California, Irvine Malaria Initiative (UCIMI) has developed gene drive-based systems for population modification of the African malaria vector mosquito, Anopheles gambiae, with the goal of contributing to the eradication of malaria. $^{27}$ Our program is concurrently conducting field research, work required before consideration of a release of GEMs, to identify candidate sites for possible confined field trials. The field research includes conducting extensive collections and analysis of local mosquito populations and engagement with stakeholders and community leaders. The UCIMI has adopted an RBM approach for engagement, applying the core concepts of the RBM to our program (Box 2).

Important to note is that the core concepts of the RBM work within established systems; they do not supersede existing and accepted laws, policy, regulation, and governance. Although application of the RBM acknowledges the importance of stakeholder and community involvement, it does not ignore existing international decision-making hierarchies or those within the country or community where it is applied. On the contrary, it complements the WHO guidance for the evaluation of GEMs by adapting to current governance, rather than replacing it. ${ }^{28}$ An $\mathrm{RBM}$ ensures that communities have a central role in directing program activities and strategies within existing systems. A research program like the UCIMI, applying an RBM to engagement, is conducted in an attempt to reduce asymmetry among the program, external experts, academics, and the recipients of the technology. Applying an RBM acknowledges that stakeholders and individuals who are considering the technology should be leading conversations that inform decisions, policies, and frameworks for the application of the technology.

Applying the RBM. The established RBM outlines specific concepts for successful implementation of the model. ${ }^{18} \mathrm{We}$ have adopted these concepts as a set of guidelines for the application of engagement, emphasizing the importance of collaboration, relationship development, and full participation of people and their leaders in malaria-endemic countries (Box 3).

Commitment to the model. Program commitment to the RBM model is critical, and this commitment should be reflected in the mission, vision, values, and actions that program leaders demonstrate with colleagues, staff, collaborators, and community members. Decision-making is decentralized in an RBM. This changes who is making decisions, moving from an entirely position-based decision-making process to one that is knowledge-based. Decentralized decision-making creates conditions in which decision-making authority is given to those who are in the best position to determine the adequacy and efficacy of the decisions being made. It often involves active participation from individuals at the point of service/intervention in the development of specific strategies, timelines, and activities.

Within this approach, researchers expand their considerations when developing a project strategy and timeline to anticipate that there will be regular adjustments and revisions, with flexibility to respond to dynamic environments. Timelines for phases of development to deployment will be determined not only by scientific feasibility but also by community and stakeholder groups driving decisions regarding engagement, regulation, and acceptance of the technology. The UCIMI program applied this concept by sharing a proposed project plan and timeline early in the initial engagement phase with stakeholders from potential field sites to address specific needs, requirements, and concerns unique to the site. From these early discussions, a final site-specific program timeline

Box 2

Relationship-based model core concepts

1. Individuals drive decisions that determine their health and health outcomes.

2. Relationships are important to understand the history, values, beliefs, and circumstances of communities and community members.

3. Relationships of trust support collaboration, shared decision-making, and active participation.

4. Optimal conditions for decision-making in the development of public health programs/interventions include the recipients at the point of care.

5. Communication, collaboration, and coordination between individuals/communities and the programs, agents, health systems, and services involved in the delivery of health and public health interventions are needed.

6. In complex, dynamic human systems, one size does not fit all. 
Box 3

Guidelines for applying the relationship-based model to community and regulatory engagement strategies

1. Commitment from program/institutional leadership to the model.

2. Team-based approach to engagement.

3. Building on existing strengths and resources.

4. Development of an environment that supports the model: communication and engagement training, safe spaces and places for open communication, and pathways for information sharing among groups.

5. Engagement planning, development, and implementation are led by site stakeholders and communities.

6. Continuous evaluation and improvement.

was determined by its stakeholders. These timeline and project work plan were then shared with existing and potential funders in conjunction with a written description of the RBM for engagement. At each stage of the project work, the work plan and timeline are revisited and reviewed with site stakeholders and partners, adjusting as needed to meet the requirements and priorities of the field site.

An RBM requires system-wide commitment, and this begins with the ability of program leadership to effectively communicate with funders and research institutions to which they are held accountable, the benefits, challenges, and philosophy behind the application of the model so that there is a shared understanding about how program goals will be met over time. Commitment from funders and research institutions is of critical importance in a model that requires flexibility in the ability of the program to respond to community feedback. Program leadership commitment to the model also will require a commitment to establishing collaborative environments that are supportive in the development of relationships of trust and respect.

Interdisciplinary approach. Community engagement. Every member of the research program is an integral part of the CE team as they come into contact with public, stakeholder, or community groups by virtue of their participation in the program. In addition to the program research team, the RBM calls for a local, site-specific interdisciplinary team that represents all entities contributing directly or indirectly to the health of the community at the field site to lead CE efforts. Applying this concept for novel malaria control strategies requires inclusion of those affected by malaria and those who are central to decision-making and determining appropriate responses to malaria control, treatment, and surveillance at the field site. Determination of the appropriate agencies and programs that need to be involved in engagement efforts should be guided by stakeholders at the field site where the research is being conducted.

The UCIMI research program initiated engagement with the Ministries of Health in the prospective field site countries to begin conversations about the research and to develop a relationship with the government agencies responsible for malaria control, and who ultimately will determine whether a field trial of GEMs with gene drive can/should be considered. This relationship has proven beneficial in connecting the program to the appropriate people in leadership roles and has provided the research program with credibility for having already passed through the required channels to allow for initiation of conversation and collaboration. In addition, this relationship assisted in providing the research program with understanding of the national health system and the important intersections between community members, the health system, and malaria-related agencies and programs who will lead the development of the site-specific engagement team. Focusing initial engagement efforts on assessing who is involved in malaria control efforts, where community members receive information about malaria, what sources of information are trusted, and how they intersect with the communities is essential in the development of the interdisciplinary engagement team.

Regulatory engagement. Just as a site-specific interdisciplinary team is important for CE, so is it important for regulatory engagement. The regulatory team, working within existing international and national regulation and policy, would determine appropriate regulation of this novel new technology. They also would determine how an assessment of risk is completed, who is involved, and if and when there is a need for external experts, international regulators, and neighboring countries to consult in the assessment and evaluation processes. Risk identification exercises can be carried out by the research team (comprising project and local collaborators), and information developed passed along to regulators for consideration in the statutory process. The regulatory team composition and participation are determined by site stakeholders and may consist of national regulators, biosafety committees, environmental health experts, policy makers, and community leaders. Potential conflicts of interest must be declared and taken into consideration when assembling the team. The role of the research program in the development of this team is to provide support, information, and resources to stakeholders who are driving decisions to ensure that the overall regulatory process reflects and respects national goals and legislation.

Build on existing strengths and resources. The RBM transforms healthcare delivery within an existing system, building on the strengths, resources, and expertise already present. How well individuals are able to engage with other professionals, sectors, agencies, and local communities will influence how trust is built, the effectiveness and coordination of functions, and how health problems and issues are defined and addressed. Malaria research programs applying the gene drive technology at a field site can learn and benefit from existing relationships and collaborations, education and awareness campaigns, social media and website access, and other tools and resources that are part of the existing malaria control programs and/or health system. In addition, understanding the existing regulations, policies, and governing agencies is a critical first step in discussions about regulation of gene drive organisms at a field site.

Recognizing and respecting the history, development, functionality, successes, and challenges of local efforts and infrastructure is critical in the development of engagement strategies. The UCIMI, in collaboration with the Ministries 
of Health at their candidate field sites, hosted workshops with stakeholders, community leaders, educators, nongovernmental organizations (NGOs), and other agencies in an effort to assess existing malaria resources and outreach activities. Through these workshops and guidance from the Ministries, existing programs and initiatives were identified as important partners and collaborators for the research program in guiding best practices and strategies for engaging local communities.

The RBM places field site stakeholders in an important position of advising how to allocate research program resources to effectively and sustainably engage communities about each phase of the research they may consider advancing. Investing resources and support to strengthen existing structures, services, and initiatives may enhance effectiveness and coordination of malaria-related functions of the larger public health and regulatory systems within the field site, particularly in early phases of engagement. For example, the UCIMI was advised by stakeholders to partner with existing malaria health education organizations in the initial phases of engagement to assess community understanding of malaria transmission and current controls being used. These partnerships offer opportunities for the program to advance their understanding of community knowledge through trusted sources, while providing resources and support to enhance existing community education efforts. In the advanced phases of engagement that require delivery of information about the technology, stakeholders will provide direction to the program regarding appropriate engagement strategies. The goal is to establish shared, as opposed to conflicting, interests.

Here again, conflict of interest is an important consideration when determining with site stakeholders how and when to partner with external resources, as opposed to existing internal resources, to support program engagement. The UCIMI works with guidance from trusted local experts and agencies to advance the ability of the research program in assessing risk and community acceptance of the technology. Application of the RBM places these local experts and agencies as drivers of these activities in an effort to minimize the possibility that the program inadvertently impacts or influences how these existing resources function and are perceived within their communities. Guidance from stakeholders is essential in addressing these challenges.

Environment for building/strengthening relationships. Developing an environment that supports the building of strong, trusting relationships among program team members, stakeholders, and community members is a foundational component of the RBM and an important responsibility of the program. Support for good communication and engagement practices begins with participatory training and capacity building for the program team and field site collaborators which strengthen team-based problem-solving and responsiveness, individual communication and engagement skills, and a shared understanding for how these skills assist in the development of relationships. Research program collaboration with expert(s) in communications and engagement is important to successfully guide the development and implementation of these opportunities for program team members and collaborators.

Relationship-based communication skills include active listening, open-ended questions, understanding individual communication styles and roles in relationships, identifying assumptions and communication roadblocks, reflecting (paraphrasing and restating both the feelings and words of the speaker), and developing shared language and definitions associated with the program. Group trainings and activities for the application of these skills help build capacity for good communication and relationship development.

Providing an environment that supports and encourages active community involvement in decision-making processes involves identifying/developing venues and mechanisms that allow people to engage comfortably in dialogue. This will require direction from stakeholders and community leaders about appropriate places, safe spaces, times, and processes to encourage active participation of community members. In addition, establishment of pathways that determine who, what, where, and when for consistent program communication and information sharing among key program components is an important part of developing an environment for relationships and the successful application of the RBM (Figure 1).

Relationship-based engagement planning, development, and implementation. In considering the who, how, what, where, and when questions that arise when developing community and regulatory engagement activities, the RBM points to the field site stakeholders and communities who will be directly impacted by the program for answers. There is active discussion among researchers, academics, regulators, and engagement experts about how communities are defined, how communities define themselves, what role the community wants to play/should play in the research, who influences the views and beliefs of the community, and how to assess, define, and determine risk. ${ }^{8,13,29-31}$ These are critically important questions for global public health interventions and specifically for a gene drive intervention that involves a field trial over a large geographical area.

Again, there are no "one-size-fits-all" answers to these complex questions. Each country considering technologies, like gene drive for malaria control, has its own unique set of demographics, resources, infrastructure, governance, and its own unique geographic and political position in the larger global framework that will influence how these questions are answered. External research teams, social scientists, regulators, and other experts may present a framework or set of guidelines and suggested definitions for thinking about these questions, but at the national level, the RBM places the stakeholders and community leaders in a position for determining the answers specific to their country. Working within an RBM, the responsibility of the research program includes sharing of knowledge and information about global and regional guidelines and frameworks and encouraging open dialogue about how the national decisions will work within them, influence them, and may require some accountability to them. Research program responsibilities also include support and resources (including external experts if requested) to encourage capacity building and provide insight, advice, and guidance on the development of site-specific engagement and regulatory processes.

Continuous evaluation and improvement. Continuous and ongoing evaluation and improvement of activities, methods, and processes is a key component of the RBM. If the model is to be effective, the program team at the point-ofservice delivery will be involved in the outcome indicators being measured ${ }^{18}$ and the process by which risk is assessed. 
The continuous improvement process within the RBM does not supersede independent assessments of program activities, rather it is established to allow the local program teams to facilitate appropriate responses to changing community needs, concerns, and priorities.

Evaluation and improvement for CE activities. The local CE team will direct what engagement outcomes will be measured and evaluated, how measures are defined, and how results from engagement will be shared with the larger community. Their direct involvement in evaluation begins with the collection of baseline data/information that will help inform the development of engagement activities. Activity outcome measures will guide and inform improvements and modifications to current and future engagement practices to improve effectiveness. Communication strategies and activities may need to be adapted to meet the needs, questions, and concerns of the community. Results also may inform the field site risk assessment processes for the program and provide valuable feedback and information to program stakeholders, funders and the broader network of researchers who are interested in engagement practices.

Evaluation and improvement for regulatory engagement activities. The WHO states that phased development and evaluation of a GEM will include continuous consideration of product safety and quality, as well as efficacy, which is consistent in the application of an RBM. ${ }^{28}$ Risk assessment activities typically begin with identification of protection goals, followed by identification of potential hazards and the construction of pathways to harm to estimate the likelihood and impact. $^{32}$ This evaluation is usually performed using qualitative and quantitative data derived from experts. As indicated previously, this process is initiated by the site team (researchers and site collaborators) and the information is provided to the statutory agencies. The agencies are expected to ask for additional information and analysis in their considerations of product safety and quality. This current and established process whereby field site regulators and experts determine how and when the risk assessment process will be conducted, what safety considerations need to be addressed, and if any outside experts and partners participate in this activity complements the RBM. As with previous activities, potential conflicts of interest are declared and weighted accordingly.

Other considerations. Funding. Early availability of funding and support from funders to conduct relationship-based engagement activities is essential for success of the program. Engagement should begin at the onset of the program, and to successfully engage with stakeholders and community members as outlined here, it is critical that a sufficient part of the program budget is allocated to these efforts over the entire course of the program.

Most funding agencies who support field-based research understand the need for flexibility, given that this type of research is prone to timeline disruptions because of both human and environmental dynamics in the system. Of great importance in application of the RBM is the establishment of shared understanding between research program leaders and funders that flexibility will be necessary regarding project goals and timelines. Establishing acceptable program-reporting mechanisms with funders which describe successes, challenges, and adjustments to activities or approach is important, as well as providing a detailed description of the RBM and the core concepts that guide program decision-making processes at each phase of research.

Capacity building. Active participation in the development of the new technologies, engagement strategies, regulatory pathways, and risk assessment processes will likely require different levels of capacity building for stakeholders and community partners. It is important to provide thoughtful consideration about where and how this training/capacity building may be conducted and who is capable of providing it so that conversations with field site stakeholders about training and capacity-building opportunities and options can happen early in the program engagement efforts. The research program may consider collaborating with regulatory and public health agencies, research institutions, and NGOs who have expertise in specific areas, advanced language or cultural competency, and/or previous experience at the prospective field site. Providing field site stakeholders with a menu of options at an early stage will help in the overall development of program timelines and activities. Capacitybuilding activities will help guarantee the continuity of transmission of important and fundamental knowledge among the participants involved in the project, guaranteeing both longterm technical capacity and sustainability and facilitating the establishment of relationships of common interest among all parties involved, which strengthens and validates the RBM.

\section{CONCLUSION}

Application of the RBM to engagement for field trials of GEMs for malaria control provides a framework for investigators who wish to establish meaningful and effective dialogue, collaboration, and relationships of trust in the communities where their research is conducted. The core concepts of the model not only reflect and complement recently published guidelines and frameworks for community and regulatory engagement but also apply previously tested and effective methods for developing a public health strategy that is determined by the community. The core concepts of the model offer a new approach to engagement by emphasizing the importance of stakeholder and community member leadership in the development and implementation of engagement strategies, definitions, and decisions. This means that conversations, debate, and decisions surrounding important questions about who to engage, how to engage, and when to engage involve stakeholders and community members from field sites where research is being conducted. It is critically important to have these voices represented where these conversations take place, and this model emphasizes this need. The RBM provides guidance for integrating field site communities in the development and implementation of novel malaria control interventions. It is our hope that consideration of this model for global public health interventions will contribute to the long-term goal of developing ethical, effective, and relationship-centered practices in engagement research.

Received July 16, 2020. Accepted for publication November 10, 2020. Published online December 21, 2020.

Acknowledgments: We are grateful to Christopher Kelsch who contributed to reviewing this manuscript and provided expert opinion on the application of the RBM. A. A. J. is a professor at Donald Bren at UCl. 
Financial support: Support for the preparation of this manuscript and the activities that contributed to it was provided by the University of California, Irvine Malaria Initiative (to A. K., G. L., E. B., J. M. M., G. D., and A. A. J.) (http://malaria.bio.uci.edu/) and by funding from the Open Philanthropy Project Fund (to G. L.) (www.openphilanthropy. org) (A20-3521-001).

Authors' addresses: Ana Kormos and Gregory C. Lanzaro, Microbiology and Immunology, University of California, Davis, CA, E-mails: amkormos@ucdavis.edu and gclanzaro@ucdavis.edu. Ethan Bier, Section of Cell and Developmental Biology, University of California, San Diego, La Jolla, CA, E-mail: ebier@ucsd.edu. George Dimopoulos, Department of Molecular Microbiology and Immunology, Malaria Research Institute (JHMRI), Bloomberg School of Public Health, Johns Hopkins University, Baltimore, MD, E-mail: gdimopo1@ jhu.edu. John M. Marshall, Division of Epidemiology and Biostatistics, School of Public Health, University of California, Berkeley, CA, E-mail: john.marshall@berkeley.edu. João Pinto, Global Health and Tropical Medicine, Instituto de Higiene e Medicina Tropical, Universidade Nova de Lisboa, Lisbon, Portugal, E-mail: jpinto@ inmt.unl.pt. Adionilde Aguiar dos Santos, Ministério da Saúde, Delegacia de Saúde Distrital de Água Grande, Cidade de São Tomé, São Tomé and Príncipe, E-mail: adiaguiar2@hotmail.com. Affane Bacar, Ministry of Health, Programme Nationale de Lutte Contre le Paludisme, Moroni, Union of the Comoros, E-mail: afanebacar@gmail.com. Herodes Sousa Pontes Sacramento Rompão, Ministério da Saúde, Programa Nacional de Luta contra o Paludismo, Centro Nacional de Endemias, Cidade de São Tomé, São Tomé and Príncipe, E-mail: herodesrompao@ gmail.com. Anthony A. James, Department of Microbiology and Molecular Genetics, University of California, Irvine, CA, and Department of Molecular Biology and Biochemistry, University of California, Irvine, CA, E-mail: aajames@uci.edu.

This is an open-access article distributed under the terms of the Creative Commons Attribution (CC-BY) License, which permits unrestricted use, distribution, and reproduction in any medium, provided the original author and source are credited.

\section{REFERENCES}

1. Hood NE, Brewer T, Jackson R, Wewers ME, 2010. Survey of community engagement in NIH-funded research. Clin Trans/ 3: 19-22.

2. Michener L, Cook J, Ahmed SM, Yonas MA, Coyne-Beasley T, Aguilar-Gaxiola S, 2012. Aligning the goals of communityengaged research: why and how academic health centers can successfully engage with communities to improve health. Acad Med 87: 285-291.

3. Carballar-Lejarazú R, James AA, 2017. Population modification of Anopheline species to control malaria transmission. Pathog Glob Health 111: 424-435.

4. Delany-Moretlwe S, Stadler J, Mayaud P, Rees H, 2011. Investing in the future: lessons learnt from communicating the results of HSV/HIV intervention trials in South Africa. Health Res Policy Syst 9 (Supp/ 1): S8.

5. MacQueen KM, Harlan SV, Slevin KW, Hannah S, Bass E, Moffet J, 2012. Stakeholder Engagement Toolkit for HIV Prevention Trials. Durham, NC: FHI360. Available at: https://www.fhi360.org/ resource/stakeholder-engagement-toolkit-hiv-prevention-trials. Accessed May 25, 2020.

6. Kolopack PA, Parsons JA, Lavery JV, 2015. What makes community engagement effective? Lessons from the eliminate dengue program in Queensland Australia. PLoS Negl Trop Dis 9: e0003713.

7. Lavery JM, Tindana PO, Scott TW, Harrington LC, Ramsey JM, Ytuarte-Nunez C, James AA, 2010. Towards a framework for community engagement in global health research. Trends Parasitol 26: 279-283.

8. Thizy D et al., 2019. Guidance on stakeholder engagement practices to inform the development of area-wide vector control methods. PLoS Negl Trop Dis 13: e0007286.

9. World Health Organization, 2016. Patient Engagement: Technical Series on Safer Primary Care. Geneva, Switzerland: WHO.

10. Clancy CM, 2011. Patient engagement in health care. Health Serv Res 46: 389-393.
11. Nakibinge S, Maher D, Katende J, Kamali A, Grosskurth H, Seeley J, 2009. Community engagement in health research: two decades of experience from a research project on HIV in rural Uganda. Trop Med Int Health 14: 190-195.

12. World Health Organization, 2017. WHO Community Engagement Framework for Quality, People-Centered and Resilient Health Services. Geneva, Switzerland: WHO.

13. National Academies of Sciences, Engineering, and Medicine, 2016. Gene Drives on the Horizon: Advancing Science, Navigating Uncertainty, and Aligning Research with Public Values, 1st edition. Washington, DC: The National Academies Press.

14. WHO Special Programme for Research and Training in Tropical Diseases, 2014. Guidance Framework for Testing of Genetically Modified Mosquitoes. Geneva, Switzerland: World Health Organization. Available at: https://apps.who.int/iris/handle/10665/ 127889. Accessed April 1, 2020.

15. Ramsey JM, Bond JG, Macotela ME, Facchinelli L, Valerio L, Brown DM, Scott TW, James AA, 2014. A regulatory structure for working with genetically-modified mosquitoes: lessons from Mexico. PLoS Negl Trop Dis 8: e2623.

16. World Health Organization, 2020. Malaria Eradication: Benefits, Future Scenarios and Feasibility. Geneva, Switzerland: WHO.

17. Balint $\mathrm{E}, 1969$. The possibilities of patient-centered medicine. $J R$ Coll Gen Pract 17: 269-276.

18. Koloroutis M, 2004. Relationship-Based Care: A Model for Transforming Practice, 1st edition. Minneapolis, MN: Creative Health Care Management.

19. Nundy S, Oswald J, 2014. Relationship-centered care: a new paradigm for population health management. Health 2: 216-219.

20. Gottlieb K, 2013. The nuka system of care: improving health through ownership and relationships. Int J Circumpolar Health 72. doi: 10.3402/ijch.v72i0.21118.

21. Johnston JM, Smith JJ, Hiratsuka VY, Dillard DA, Szafran QN, Driscoll DL, 2013. Tribal implementation of a patient-centred medical home model in Alaska accompanied by decreased hospital use. Int J Circumpolar Health 72. doi: 10.3402/ ijch.v72i0.20960.

22. Platchek T, Rebitzer R, Zulman D, Milstein A, 2014. Better health, less spending: Stanford University's clinical excellence research center. Health Manag Policy Innovation 2: 10-17.

23. Milstein A, Gilbertson E, 2009. American medical home runs. Health Aff (Millwood) 28: 1317-1326.

24. Clever SL, Ford DE, Rubenstein LV, Rost KM, Meredith LS, Sherbourne CD, Wang N, Arbelaez JJ, Cooper LA, 2006. Primary care patients' involvement in decision-making is associated with improvement in depression. Med Care 44: 398-405.

25. Weiner SJ, Schwartz A, Sharma G, Binns-Calvey A, Ashley N, Kelly B, Dayal A, Patel S, Weaver FM, Harris I, 2013. Patientcentered decision-making and health care outcomes. Ann Intern Med 158: 573-579.

26. Gluyas H, 2015. Patient-centred care: improving healthcare outcomes. Nurs Stand 30: 50-57.

27. Carballar-Lejarazú R, Ogaugwu C, Tushar T, Kelsey A, Pham TB, Murphy J, Schmidt H, Lee Y, Lanzaro GC, James AA, 2020. Next-generation gene drive for population modification of the malaria vector mosquito, Anopheles gambiae. Proc Natl Acad Sci U S A 117: 22805-22814.

28. World Health Organization, 2020. Evaluation of Genetically Modified Mosquitoes for the Control of Vector-Borne Diseases. Geneva, Switzerland: WHO.

29. Hartley $S$ et al., 2019. Knowledge engagement in gene drive research for malaria control. PLoS Negl Trop Dis 13: e0007233.

30. James $S$ et al., 2018. Pathway to deployment of gene drive mosquitoes as a potential biocontrol tool for elimination of malaria in sub-Saharan Africa: recommendations of a scientific working group. Am J Trop Med Hyg 98 (Supp/ 6): 1-49.

31. AUDA-NEPAD, 2018. Gene Drives for Malaria Control and Elimination in Africa. Available at: https://www.nepad.org/publication/ gene-drives-malaria-control-and-elimination-africa-0. Accessed June 1, 2019.

32. Roberts A, Andrade PP, Okumu F, Quemada H, Savadogo M, Singh JA, James S, 2017. Results from the workshop "problem formulation for the use of gene drive in mosquitoes". Am J Trop Med Hyg 96: 530-533. 